\title{
Armazenamento refrigerado de ameixas ‘Laetitia’ com uso de 1-MCP e indução de perda de massa fresca
}

\author{
Cold storage of 'Laetitia' plums treated with 1-MCP and induced to loss of fresh mass
}

\author{
Erlani de Oliveira Alves ${ }^{\mathrm{I}}$ Cristiano André Steffens ${ }^{\mathrm{I} *}$ Cassandro Vidal Talamini do Amarante ${ }^{\mathrm{I}}$ \\ Anderson Weber ${ }^{I I}$ Aquidauana MiquelotoI Auri Brackmann"
}

RESUMO

O objetivo deste trabalho foi avaliar os efeitos da temperatura de armazenamento, do uso de 1metilciclopropeno (1-MCP) e da indução de perda de massa fresca (IPMF) na qualidade de ameixas 'Laetitia'. Os tratamentos utilizados foram: $-0,5^{\circ} \mathrm{C} ; 0,5^{\circ} \mathrm{C} ; 0,5^{\circ} \mathrm{C} \mathrm{em}$ combinação com a indução de perda de massa fresca (IPMF); e 0,5 $5^{\circ} \mathrm{C}$ após tratamento com aplicação de $1-\mathrm{MCP}\left(1,0 \mu \mathrm{L} \mathrm{L}^{-1}\right)$. Frutos armazenados a $0,5^{\circ} \mathrm{C}$ e tratados com 1-MCP apresentaram os menores valores da taxa respiratória e da taxa de produção de etileno após quatro dias de exposição em condição ambiente $\left(23^{\circ} \mathrm{C} / 60 \%\right.$ de UR). Na saída da câmara fria não houve diferença entre tratamentos. Os frutos armazenados por 30 dias a $0,5^{\circ} \mathrm{C}$, em combinação com a IPMF ou 1-MCP, apresentaram na saída da câmara maior firmeza de polpa. Porém, após quatro dias, apenas os frutos armazenados a $0,5^{\circ} \mathrm{C}$ e tratados com 1-MCP apresentaram maior firmeza de polpa. $O$ índice de cor vermelha, na saida da câmara, foi menor nos frutos armazenados a $-0,5^{\circ} \mathrm{C}$ e a $0,5^{\circ} \mathrm{C}$ após tratamento com 1-MCP, não sendo observada diferença entre os tratamentos após quatro dias de exposição dos frutos em temperatura ambiente. $O$ armazenamento a $-0,5^{\circ} \mathrm{C}$ proporcionou melhor preservação dos atributos de textura dos frutos do que a $0,5^{\circ} \mathrm{C}$, e nesta temperatura o tratamento com 1-MCP proporcionou os melhores resultados. O armazenamento a $0,5^{\circ} \mathrm{C}$ em relação a $-0,5^{\circ} \mathrm{C}$ causou incremento na incidência de degenerescência da polpa após quatro dias de exposição dos frutos em condição ambiente. A melhor temperatura para $o$ armazenamento refrigerado de ameixas 'Laetitia' é de $-0,5^{\circ} \mathrm{C}$. O uso do 1-MCP e a IPMF contribuem para a manutenção da qualidade dos frutos durante o armazenamento, e o efeito do 1-MCP persiste após o armazenamento.

Palavras-chave: Prunus salicina, pós-colheita, etileno, respiração, degenerescência da polpa, qualidade.

\section{ABSTRACT}

This research was carried out to study the effects of storage temperature, treatment with 1-methycyclopropene (1$M C P$ ), and induction of fresh mass loss (IFML) on quality of 'Laetitia' plums. Fruit were treated by storage at: $-0.5^{\circ} \mathrm{C} ; 0.5^{\circ} \mathrm{C}$; $0.5^{\circ} \mathrm{C}$ combined with IFML; and at $0.5^{\circ} \mathrm{C}$ after treatment with $1-M C P\left(1.0 \mu L L^{-1}\right)$. Fruits stored at $0.5^{\circ} \mathrm{C}$ after treatment with 1-MCP exhibit the lowest respiration and ethylene production rates after four days of shelf life $\left(23^{\circ} \mathrm{C} / 60 \% \mathrm{RH}\right)$, despite of non significant difference between treatments at removal from cold storage. Fruits stored for 30 days at $0.5^{\circ} \mathrm{C}$, combined with IFML or 1-MCP, exhibit the highest flesh firmness at removal from cold storage. However, after four days of shelf life, only fruits treated with 1-MCP before storage at $0.5^{\circ} \mathrm{C}$ had the highest flesh firmness. Fruits stored at $-0.5^{\circ} \mathrm{C}$ and fruit treated with 1 $M C P$ before storage at $0.5^{\circ} \mathrm{C}$ exhibit the lowest red color development at removal from cold storage, but not after four days of shelf life. Fruits stored at $-0.5^{\circ} \mathrm{C}$ had a better preservation of texture attributes than those stored at $0.5^{\circ} \mathrm{C}$, but fruit stored at $0.5^{\circ} \mathrm{C}$ after treatment with 1-MCP had the best texture. Fruits stored at $0.5^{\circ} \mathrm{C}$ exhibit increased incidence of flesh browning after four days of shelf life in comparison to fruit stored at $-0.5^{\circ} \mathrm{C}$. The best temperature for refrigerated storage of 'Laetitia' plums is $-0.5^{\circ} \mathrm{C}$. The treatments with 1-MCP and IFML contribute to preserve fruit quality during cold storage, but only the effect of 1-MCP persist after removal from cold storage.

Key words: Prunus salicina, postharvest, ethylene, respiration, flesh browning, quality.

\section{INTRODUÇÃO}

A ameixa 'Laetitia', por possuir um rápido amadurecimento, apresenta curta vida pós-colheita e

IDepartamento de Agronomia, Universidade do Estado de Santa Catarina (UDESC). Av. Luiz de Camões, 2090, Bairro Conta Dinheiro, 88520-000, Lages, SC, Brasil. E-mail: steffens@cav.udesc.br.*Autor para correspondência.

IIDepartamento de Fitotecnia, Universidade Federal de Santa Maria (UFSM), Santa Maria, RS, Brasil. 
rápida perda de qualidade, limitando a receita do produtor devido ao baixo valor obtido no período de safra. Contudo, a redução de perdas e o prolongamento do período de oferta podem ser obtidos por meio da otimização das condições de armazenamento refrigerado, possibilitando ao produtor comercializar o produto durante a entressafra e obter uma melhor remuneração pela sua produção.

A utilização do armazenamento refrigerado consiste em uma excelente alternativa para retardar o amadurecimento e prolongar a vida pós-colheita de ameixas. A redução da temperatura constitui o principal fator que influencia a manutenção da qualidade dos frutos durante o armazenamento (STEFFENS et al., 2007). Segundo BRACKMANN et al. (2001), o armazenamento refrigerado é um dos principais métodos utilizados para conservação de ameixas. Porém, no Brasil, não há conhecimento da temperatura ideal para o armazenamento da ameixa 'Laetitia'.

Outras técnicas podem ser utilizadas para complementar o efeito da refrigeração na manutenção da qualidade dos frutos, como a utilização do 1metilciclopropeno (1-MCP) (WATKINS, 2006) e a indução de perda de massa fresca (IPMF) (BRACKMANN et al., 2007). Vários trabalhos têm demonstrado o efeito do 1-MCP sobre o retardo no amadurecimento de frutos (DONG etal., 2002;ARGENTA et al., 2003; VALERO et al., 2004; CANDAN et al., 2006; MANGANARIS et al., 2008).

O processo de transpiração durante o armazenamento dos frutos é a causa principal da perda de massa fresca. A perda de massa por transpiração não implica somente a perda de massa comercializável, mas também a perda de qualidade do produto (BRACKMANN et al., 2007). Não há trabalhos de pesquisa que tentam elucidar a IPMF como alternativa para manter a qualidade da conservação pós-colheita em ameixa. A utilização da IPMF apresentou efeito positivo no controle do amadurecimento de maçãs 'Gala', além de reduzir a incidência de podridões e de distúrbios fisiológicos (BRACKMANN et al., 2007). Sendo assim, o armazenamento refrigerado de ameixas 'Laetitia' em uma temperatura adequada, associado ao uso do 1-MCP ou da IPMF, pode aumentar o período de conservação dos frutos.

O objetivo deste trabalho foi avaliar os efeitos da temperatura de armazenamento e do uso de 1-MCP e da IPMF sobre a conservação da qualidade de ameixas 'Laetitia'.

\section{MATERIAL E MÉTODOS}

Ameixas 'Laetitia' for am colhidas em pomar comercial, localizado no município de Lages, Santa Catarina (SC), e transportadas para o Laboratório do Núcleo de Pesquisa e Pós-Colheita - NPP, da Universidade Federal de Santa Maria, localizado no município de Santa Maria, Rio Grande do Sul (RS). No laboratório, os frutos foram selecionados, sendo eliminandos aqueles com lesões, defeitos, ferimentos ou dano mecânico. Posteriormente, procedeu-se à homogeneização das unidades experimentais.

O delineamento experimental adotado foi o inteiramente casualizado, com cinco repetições por tratamento, sendo a unidade experimental composta por 30 frutos. Os tratamentos utilizados foram armazenados a: $-0,5^{\circ} \mathrm{C} ; 0,5^{\circ} \mathrm{C} ; 0,5^{\circ} \mathrm{C}$ em combinação com a indução de perda de massa fresca; e $0,5^{\circ} \mathrm{C}$, após tratamento com 1-MCP $\left(1,0 \mu \mathrm{L} \mathrm{L}^{-1}\right)$.

Os frutos de todos os tratamentos foram armazenados em minicâmaras experimentais, com capacidade de 180L. A IPMF foi provocada de forma constante, através da absorção da umidade do ar da minicâmara. Esse procedimento foi efetuado com uma bomba de membrana que permitia circular o ar do interior da minicâmara para dentro de um recipiente contendo sílica gel, conforme metodologia descrita por BRACKMANN et al. (2007). Diariamente, a bomba foi ligada para absorver $5,7 \mathrm{~g}$ de água da minicâmara, atingindo, ao final do armazenamento, $171 \mathrm{~g}$ de água absorvida, representando $1,5 \%$ de perda de massa fresca das amostras.

Para o tratamento com 1-MCP, foi utilizado o produto SmartFresh $^{\circledR}(0,14 \%$ de 1 -MCP na formulação pó), na relação de $0,16 \mathrm{~g}$ de produto $\mathrm{m}^{-3}$ de câmara, para obter $1,0 \mu \mathrm{L} \mathrm{L}^{-1}$ de 1 -MCP. O produto foi colocado em frasco hermético e solubilizado em água, na temperatura ambiente. Posteriormente, o frasco foi introduzido na minicâmara, e a solução foi transferida para uma placa de Petri, por meio da abertura lateral, e vedada imediatamente. Os frutos ficaram expostos ao tratamento durante 24 horas, antes do armazenamento a $0,5^{\circ} \mathrm{C}$.

Após 30 dias de armazenamento, os frutos foram transportados para o Laboratório de Pesquisa em Fisiologia e Tecnologia de Pós-Colheita, da Universidade do Estado de Santa Catarina (UDESC), em Lages, SC, onde foram feitas as análises de qualidade, na saída da câmara e após quatro dias de exposição dos frutos em condição ambiente $\left(23^{\circ} \mathrm{C} / 60 \%\right.$ de UR). As variáveis analisadas foram: taxa respiratória e taxa de produção de etileno, firmeza de polpa, teor de sólidos solúveis (SS), acidez titulável (AT), índice de cor vermelha (ICV), incidência e índice de 
degenerescência da polpa, incidência de podridões e atributos de textura.

As taxas respiratória e de produção de etileno foram quantificadas, colocando-se 15 frutos de cada amostra em um recipiente com o volume de $2,3 \mathrm{~L}$, com fechamento hermético. A taxa respiratória foi obtida pela diferença da concentração de $\mathrm{CO}_{2}$ no interior do recipiente, imediatamente após o seu fechamento e depois de uma hora do acondicionamento dos frutos. Alíquotas de gás $(1000 \mu \mathrm{L})$ foram retiradas dos recipientes por meio de um septo e injetadas em um cromatógrafo a gás Varian $^{\circledR}$, modelo CP-3800, equipado com uma coluna Porapak $N^{\circledR}$ de $3 \mathrm{~m}$ de comprimento (80-100 mesh), metanador e detector de ionização de chama. As temperaturas da coluna, do detector, do metanador e do injetor foram de $45^{\circ} \mathrm{C}, 120^{\circ} \mathrm{C}, 300^{\circ} \mathrm{C}$ e $110^{\circ} \mathrm{C}$, respectivamente. Os fluxos de nitrogênio, hidrogênio e ar sintético foram de 70, 30 e $300 \mathrm{~mL} \mathrm{~min}^{-1}$, respectivamente. Os valores da atividade respiratória e da taxa de produção de etileno foram expressos em nmol de $\mathrm{CO}_{2} \mathrm{~kg}^{-1} \mathrm{~s}^{-1}$ e pmol de $\mathrm{C}_{2} \mathrm{H}_{4} \mathrm{~kg}^{-1} \mathrm{~s}^{-1}$, respectivamente.

$O$ índice de cor vermelha foi determinado avaliando-se a superfície dos frutos recoberta com coloração vermelha, sendo atribuídas notas de 1 a 4 (1, 2,3 e 4 para o fruto com, respectivamente, $0-25 \%, 26$ $50 \%, 51-75 \%$ e $76-100 \%$ da superfície pigmentada de vermelho). $\mathrm{O}$ índice foi calculado pelo somatório dos produtos do número de frutos pela respectiva nota $\mathrm{e}$ pelo respectivo nível, dividido pelo total de frutos da amostra.

A firmeza de polpa foi determinada em dois pontos na região equatorial dos frutos, em lados opostos, após remoção de uma pequena porção da epiderme, com o auxílio de um penetrômetro equipado com ponteira de $8 \mathrm{~mm}$ de diâmetro.

Os atributos de textura foram analisados com um texturômetro eletrônico TAXT-plus ${ }^{\circledR}$ (Stable Micro Systems Ltd., Reino Unido), em termos de força necessária para o rompimento da epiderme e de força para a penetração na polpa e para a compressão do fruto inteiro. Para a quantificação da força necessária para o rompimento da epiderme e para a penetração na polpa, foi utilizada ponteira modelo PS2, com $2 \mathrm{~mm}$ de diâmetro, a qual foi introduzida na polpa a uma profundidade de $5 \mathrm{~mm}$, com velocidades pré-teste, teste e pós-teste de 30,5 e $30 \mathrm{~mm} \mathrm{~s}^{-1}$, respectivamente. A resistência do fruto à compressão foi determinada usando-se uma plataforma plana, modelo $\mathrm{P} / 75$, com $75 \mathrm{~mm}$ de diâmetro, que exerceu uma força de compressão até uma deformação de $5 \mathrm{~mm}$ na superfície do fruto.
Os valores de acidez titulável (AT; meq $100 \mathrm{~mL}^{-1}$ ) foram obtidos em amostra de $10 \mathrm{~mL}$ de suco, de fatias transversais, retiradas da região equatorial dos frutos submetidas à extração em uma centrífuga. A amostra obtida foi diluída em $90 \mathrm{~mL}$ de água destilada e titulada com solução de $\mathrm{NaOH} 0,1 \mathrm{~N}$ até $\mathrm{pH} 8,1$. Os teores de SS ( ${ }^{\circ}$ Brix) foram determinados por refratometria, utilizando-se o suco extraído conforme descrito para a AT, sendo realizada a correção do efeito da temperatura para $20^{\circ} \mathrm{C}$.

A incidência de degenerescência da polpa (\%) foi avaliada por meio de corte transversal na região equatorial do fruto, sendo contabilizados os frutos que apresentaram escurecimento da polpa. $\mathrm{O}$ índice de degenerescência da polpa foi avaliado por meio de notas (1-3) atribuídas aos frutos, sendo 1 ausência de degenerescência, 2 com até $50 \%$ da polpa afetada e 3 acima de $50 \%$ da polpa afetada.

Antes da aplicação dos tratamentos, duas amostras de 15 frutos foram analisadas para determinar as características da qualidade inicial das ameixas, as quais apresentaram firmeza de polpa de $42,0 \mathrm{~N}$, teor de SS de $9,3^{\circ}$ Brix eAT de $31,2 \mathrm{meq} 100 \mathrm{~mL}^{-1}$.

Os dados foram submetidos à análise da variância (ANOVA), sendo os dados em porcentagem transformados pela fórmula $\operatorname{arcsen} \sqrt{x / 100}$ antes de serem submetidos à ANOVA. As médias de tratamentos foram comparadas pelo teste de Tukey, a 5\% de probabilidade de erro.

\section{RESULTADOS E DISCUSSÃO}

As taxas respiratória e de produção de etileno não diferiram entre tratamentos na saída da câmara, porém, após quatro dias de exposição à condição ambiente, os frutos armazenados na temperatura de $0,5^{\circ} \mathrm{C}$ e tratados com $1-\mathrm{MCP}$ apresentaram as menores taxas respiratória e de produção de etileno (Tabela 1).

Estudos realizados com ameixas demonstraram que o 1-MCP reduz consideravelmente a atividade respiratória e a produção de etileno (DONG et al., 2002; ARGENTA et al., 2003), resultados confirmados no presente trabalho. A menor taxa de produção de etileno em frutos tratados com 1-MCP deve-se ao seu efeito em reduzir a atividade das enzimas sintase ACC e oxidase ACC, que são consideradas os pontos de controle da biossíntese de etileno (WATKINS, 2006). Com relação ao efeito da IPMF, em maçãs 'Gala', BRACKMANN et al. (2007) obtiveram menores valores de taxas respiratória e de produção de etileno, porém este resultado não foi obtido na ameixa 'Laetitia' no presente trabalho. 
Alves et al

Tabela 1 - Taxas respiratórias e de produção de etileno em ameixa 'Laetitia' após 30 dias de armazenamento sob refrigeração e mais quatro dias em condição ambiente $\left(20 \pm 2^{\circ} \mathrm{C} / 60 \pm 5 \%\right)$. Lages, 2008.

\begin{tabular}{|c|c|c|c|c|}
\hline \multirow{2}{*}{ Tratamento } & \multicolumn{2}{|c|}{-------Taxa respiratória $\left(\mathrm{nmol} \mathrm{CO}_{2} \mathrm{~kg}^{-1} \mathrm{~s}^{-1}\right)$------ } & \multicolumn{2}{|c|}{----Taxa de produção de etileno $\left(\mathrm{pmol} \mathrm{kg}^{-1} \mathrm{~s}^{-1}\right)$} \\
\hline & Saída da câmara & $\begin{array}{l}\text { Quatro dias em condição } \\
\text { ambiente }\end{array}$ & Saída da câmara & $\begin{array}{l}\text { Quatro dias em condição } \\
\text { ambiente }\end{array}$ \\
\hline$-0,5^{\circ} \mathrm{C}$ & $232,4 \mathrm{a}$ & $236,1 \mathrm{a}$ & $11,1 \mathrm{a}$ & $333,6 \mathrm{a}$ \\
\hline $0,5^{\circ} \mathrm{C}$ & $330,4 a$ & $269,2 \mathrm{a}$ & $59,7 \mathrm{a}$ & $178,9 \mathrm{a}$ \\
\hline $0,5^{\circ} \mathrm{C}+\mathrm{IPMF}^{*}$ & $99,5 \mathrm{a}$ & $262,6 a$ & $67,8 \mathrm{a}$ & $266,9 \mathrm{a}$ \\
\hline $0,5^{\circ} \mathrm{C}+1-\mathrm{MCP}$ & $234,8 \mathrm{a}$ & $153,9 b$ & $2,40 \mathrm{a}$ & $11,2 b$ \\
\hline CV $(\%)$ & 16,70 & 4,27 & 57,60 & 14,70 \\
\hline
\end{tabular}

Médias nas colunas não seguidas pela mesma letra diferem pelo teste de Tukey, a 5\% de probabilidade de erro.

* Indução de perda de massa fresca.

Os frutos armazenados a $0,5^{\circ} \mathrm{C}$, em combinação com IPMF ou 1-MCP, na saída da câmara, apresentaram maior firmeza de polpa (Tabela 2). A maior firmeza de polpa observada na saída da câmara, no tratamento com indução de perda de massa fresca, também foi observado em maçãs 'Gala' (BRACKMANN et al., 2007). Porém, após os quatro dias em condição ambiente, apenas os frutos armazenados a $0,5^{\circ} \mathrm{C}$ e tratados com 1-MCP apresentaram maior firmeza de polpa (Tabela 2). A resposta da firmeza de polpa ao 1-MCP está diretamente relacionada ao seu efeito sobre a redução na ação de etileno, pois o etileno é necessário para a promoção na atividade de enzimas responsáveis pelo amolecimento dos frutos (JOHNSTON et al., 2001; MAJUMDER \& MAZUMDAR, 2002). Ameixas tratadas com 1-MCP apresentaram menor taxa de produção de etileno (Tabela 1), resultando assim em maior firmeza de polpa. O efeito do 1-MCP sobre o retardo na perda da firmeza de polpa em ameixas também foi reportado por outros autores (DONG et al., 2001; ARGENTA et al., 2003; KHAN \& SING, 2005; CANDAN et al., 2006; MANGANARIS et al., 2008).

Os teores de sólidos solúveis não foram influenciados pelos tratamentos, tanto na saída câmara, como após quatro dias de exposição dos frutos em temperatura ambiente (Tabela 2). BRACKMANN et al. (2001) também não encontraram diferenças nos teores de SS em ameixas 'Pluma 7' e 'Reubennnel' armazenadas em diferentes temperaturas. Em maçãs 'Gala', os teores de SS apresentaram-se maiores nos frutos armazenados com IPMF (BRACKMANN et al., 2007), entretanto, este

Tabela 2 - Qualidade físico-química em ameixa 'Laetitia' após 30 dias de armazenamento sob refrigeração e mais quatro dias em condição ambiente $\left(20 \pm 2^{\circ} \mathrm{C} / 60 \pm 5 \%\right)$. Lages, 2008 .

\begin{tabular}{|c|c|c|c|c|c|c|}
\hline \multirow{2}{*}{ Tratamentos } & \multirow{2}{*}{$\begin{array}{c}\text { Firmeza de polpa } \\
\text { (N) }\end{array}$} & \multirow{2}{*}{$\begin{array}{c}\text { Sólidos solúveis } \\
\left({ }^{\circ} \text { Brix }\right)\end{array}$} & \multirow{2}{*}{$\begin{array}{l}\text { Acidez titulável } \\
\left(\mathrm{meq} 100 \mathrm{~mL}^{-1}\right)\end{array}$} & \multirow{2}{*}{$\mathrm{ICV}^{* *}(1-4)$} & \multicolumn{2}{|c|}{------Degenerescência da polpa------ } \\
\hline & & & & & Incidência (\%) & Índice $(1-3)^{* * *}$ \\
\hline \multicolumn{7}{|c|}{ 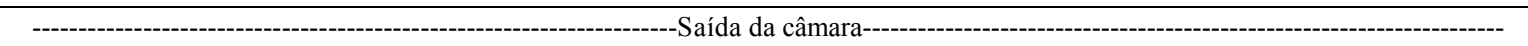 } \\
\hline$-0,5^{\circ} \mathrm{C}$ & $40,7 \mathrm{~b}$ & $9,10 \mathrm{a}$ & $23,1 \mathrm{a}$ & $3,10 \mathrm{bc}$ & $1,65 \mathrm{a}$ & $1,05 \mathrm{a}$ \\
\hline $0,5^{\circ} \mathrm{C}$ & $27,0 \mathrm{c}$ & $9,33 \mathrm{a}$ & $22,3 a$ & $3,67 \mathrm{a}$ & $1,65 \mathrm{a}$ & $1,05 \mathrm{a}$ \\
\hline $0,5^{\circ} \mathrm{C}+\mathrm{IPMF}^{*}$ & $47,8 \mathrm{a}$ & $9,10 \mathrm{a}$ & $20,6 a$ & $3,37 \mathrm{ab}$ & $0,82 \mathrm{a}$ & $1,01 \mathrm{a}$ \\
\hline $0,5^{\circ} \mathrm{C}+1-\mathrm{MCP}$ & $42,7 \mathrm{ab}$ & $9,00 \mathrm{a}$ & $25,3 \mathrm{a}$ & $2,76 \mathrm{c}$ & $1,65 \mathrm{a}$ & $1,03 \mathrm{a}$ \\
\hline $\mathrm{CV}(\%)$ & 7,18 & 3,14 & 6,30 & 5,96 & 76,00 & 5,11 \\
\hline \multicolumn{7}{|c|}{ 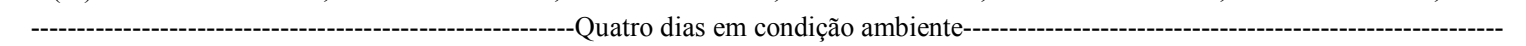 } \\
\hline$-0,5^{\circ} \mathrm{C}$ & $9,69 \mathrm{c}$ & $8,25 \mathrm{a}$ & $17,2 b$ & $4,00 \mathrm{a}$ & $1,65 b$ & $1,03 b$ \\
\hline $0,5^{\circ} \mathrm{C}$ & $9,39 \mathrm{c}$ & $8,40 \mathrm{a}$ & $18,9 \mathrm{~b}$ & $3,95 \mathrm{a}$ & $4,15 \mathrm{a}$ & $1,15 \mathrm{a}$ \\
\hline $0,5^{\circ} \mathrm{C}+\mathrm{IPMF}^{*}$ & $15,3 b$ & $8,35 \mathrm{a}$ & $19,0 \mathrm{~b}$ & $3,93 \mathrm{a}$ & $0,82 b$ & $1,00 \mathrm{~b}$ \\
\hline $0,5^{\circ} \mathrm{C}+1-\mathrm{MCP}$ & $23,2 \mathrm{a}$ & $8,40 \mathrm{a}$ & $22,7 \mathrm{a}$ & $3,98 \mathrm{a}$ & $1,65 b$ & $1,02 b$ \\
\hline $\mathrm{CV}(\%)$ & 9,60 & 6,90 & 5,20 & 1,30 & 45,00 & 4,68 \\
\hline
\end{tabular}

Médias nas colunas não seguidas pela mesma letra diferem pelo teste de Tukey, a 5\% de probabilidade de erro.

*Indução por perda de massa fresca.

**Índice de cor da vermelha: $1=0-25 \% ; 2=26-50 \% ; 3=51-75 \% ; 4=$ acima de $75 \%$ da superfície recoberta com cor vermelha.

***Índice de degenerescência da polpa: $1=$ ausência; $2=$ até $50 \%$ da polpa afetada; $3=$ acima de $50 \%$ da polpa afetada. 
mesmo efeito não foi observado no presente trabalho. Com relação ao 1-MCP, outros trabalhos relatam que o 1-MCP não influencia os teores de SS em ameixas 'Laetitia'(ARGENTAet al., 2003), 'Fortune', 'Angeleno', 'President' (MENNITI et al., 2004) e 'Blackamber' (CANDAN et al., 2006).

A acidez titulável não apresentou diferença estatística entre tratamentos na saída da câmara (Tabela 2). Porém, na avaliação realizada após quatro dias em temperatura ambiente, os valores de AT foram maiores nos frutos armazenados a $0,5^{\circ} \mathrm{C}$, após tratamento com 1-MCP (Tabela 2). ARGENTA et al. (2003) também observaram maior AT em ameixas 'Laetitia' tratadas com 1-MCP. Esse efeito do 1-MCP sobre a manutenção da AT dos frutos pode estar relacionado ao menor consumo dos ácidos no ciclo dos ácidos tricarboxílicos, pela redução na atividade respiratória (Tabela 1).

$\mathrm{O}$ índice de cor vermelha na saída da câmara foi menor nos frutos armazenados $-0,5^{\circ} \mathrm{C}$ e a $0,5^{\circ} \mathrm{C}$ após tratamento com 1-MCP, em relação aos frutos armazenados a $0,5^{\circ} \mathrm{C}$ e sem prévia aplicação de $1-\mathrm{MCP}$ (Tabela 2). O efeito da baixa temperatura e do 1-MCP sobre o índice de cor vermelha deve-se provavelmente à menor degradação da clorofila e síntese de carotenóides e antocianinas. A mudança na cor durante o amadurecimento de ameixas é um processo dependente de etileno (ARGENTA et al., 2003), o que explica o menor valor de índice de coloração vermelha em frutos tratados com 1-MCP. Porém, após quatro dias de exposição dos frutos em temperatura ambiente, mesmo com valores de etileno bastante reduzidos, observou-se uma rápida evolução da coloração vermelha em todos os tratamentos, não sendo possível observar diferenças entre tratamentos (Tabela 2). Pode ser um indicativo de que o etileno não está diretamente e proporcionalmente relacionado com a mudança de coloração. DONG et al. (2002) concluiu que o etileno não é necessário para o desenvolvimento da cor vermelha, por meio de experimentos com frutos de damasco e ameixas.

Não houve diferença entre tratamentos quanto à incidência e ao índice de degenerescência da polpa, na análise realizada na saída da câmara (Tabela 2). Contudo, após quatro dias de exposição dos frutos em condição ambiente, os maiores valores de incidência e índice de degenerescência da polpa foram observados nos frutos armazenados a $0,5^{\circ} \mathrm{C}$ (Tabela 2). Isso demonstra que a temperatura de $-0,5^{\circ} \mathrm{C}$ foi mais adequada para o armazenamento refrigerado de ameixas 'Laetitia' durante 30 dias e que, na temperatura de $0,5^{\circ} \mathrm{C}$, a aplicação de 1-MCP e a indução de perda de massa fresca contribuem para a redução desse distúrbio fisiológico. BRACKMANN et al. (2007) observaram menor ocorrência de degenerescência da polpa em maçãs 'Gala' com a IPMF, concordando com os resultados obtidos no presente trabalho. O efeito do 1MCP na redução da incidência de degenerescência da polpa em ameixas 'Laetitia' também foi observado por ARGENTA et al. (2003).

Observou-se que a temperatura $0,5^{\circ} \mathrm{C}$, com aplicação do 1-MCP, proporcionou frutos com maiores valores para os atributos de força para ruptura da epiderme e penetração da polpa, tanto na saída da câmara, quanto após quatro dias em temperatura ambiente. Para o atributo resistência do fruto à compressão, na saída da câmara, não houve diferenças entre as temperaturas de $-0,5^{\circ} \mathrm{C}$ e $0,5^{\circ} \mathrm{C}$ com aplicação do 1-MCP. Porém, após quatro dias em temperatura ambiente, o maior valor foi em ameixas armazenadas a $0,5^{\circ} \mathrm{C}$ com aplicação do 1-MCP (Tabela 3). O 1-MCP

Tabela 3 - Atributos de textura em ameixa 'Laetitia' após 30 dias de armazenamento sob refrigeração e mais quatro dias em condição ambiente $\left(20 \pm 2^{\circ} \mathrm{C} / 60 \pm 5 \%\right.$ de UR). Lages, 2008 .

\begin{tabular}{|c|c|c|c|}
\hline Tratamentos & Força para ruptura da epiderme $(\mathrm{N})$ & Força para penetração da polpa (N) & Resistência do fruto à compressão $(\mathrm{N})$ \\
\hline$-0,5^{\circ} \mathrm{C}$ & $8,64 b$ & ída da câmara----- & $83,5 \mathrm{a}$ \\
\hline $0,5^{\circ} \mathrm{C}$ & $6,61 \mathrm{c}$ & $1,22 \mathrm{c}$ & $48,6 b$ \\
\hline $0,5^{\circ} \mathrm{C}+\mathrm{IPMF}^{*}$ & $6,51 \mathrm{c}$ & $1,36 \mathrm{c}$ & $52,4 \mathrm{~b}$ \\
\hline $0,5^{\circ} \mathrm{C}+1-\mathrm{MCP}$ & $10,3 \mathrm{a}$ & $2,44 \mathrm{a}$ & $80,9 \mathrm{a}$ \\
\hline $\mathrm{CV}(\%)$ & 8,24 & 14,41 & 12,57 \\
\hline$-0,5^{\circ} \mathrm{C}$ & $4,96 b$ & $0,62 b$ & $35,8 b$ \\
\hline $0,5^{\circ} \mathrm{C}$ & $5,20 \mathrm{~b}$ & $0,80 \mathrm{~b}$ & $29,0 \mathrm{c}$ \\
\hline $0,5^{\circ} \mathrm{C}+\mathrm{IPMF}$ & $4,72 b$ & $0,63 b$ & $34,4 b$ \\
\hline $0,5^{\circ} \mathrm{C}+1-\mathrm{MCP}$ & $8,23 \mathrm{a}$ & $1,20 \mathrm{a}$ & $52,4 \mathrm{a}$ \\
\hline $\mathrm{CV}(\%)$ & 6,79 & 16,37 & 4,10 \\
\hline
\end{tabular}

Médias nas colunas não seguidas pela mesma letra diferem pelo teste de Tukey, a 5\% de probabilidade de erro.

* Indução de perda de massa fresca. 
inibe a indução do amadurecimento do fruto, mantendo uma maior consistência da polpa (ARGENTA et al., 2003; VALERO et al., 2003; CANDAN et al., 2006; MANGANARIS et al., 2008).

\section{CONCLUSÕES}

A melhor temperatura para o armazenamento refrigerado de ameixas 'Laetitia' por 30 dias é de $-0,5^{\circ} \mathrm{C}$. Em ameixas 'Laetitia' armazenadas a $0,5^{\circ} \mathrm{C}$, o uso do 1MCP e da indução de perda de massa fresca contribui para retardar o amadurecimento e reduz a degenerescência, e o efeito do 1-MCP sobre a manutenção da firmeza se mantém após exposição dos frutos em temperatura ambiente.

\section{AGRADECIMENTOS}

Os autores agradecem à Coordenação de Aperfeiçoamento de Pessoal de Nível Superior (CAPES), e à Fundação de Amparo à Pesquisa e Desenvolvimento Científico e Tecnológico de Santa Catarina (FAPESC), pelo apoio financeiro a este projeto.

\section{REFERÊNCIAS}

ARGENTA, L.C. et al. Ripening and quality of 'Laetitia' plums following harvest and cold storage as affected by inhibition of ethylene action. Pesquisa Agropecuária Brasileira, Brasília, v.38, n.10, p.1139-1148, 2003. Disponível em: <http:// www.scielo.br/ scielo.php? script $=$ sci arttext\&pid $=\mathrm{S} 0100$ $204 X 2003001000002 \& \operatorname{lng}=\mathrm{en} \& \mathrm{nrm}=\mathrm{isso}>$. Acesso em: 22 set. 2009. doi: 10.1590/S0100-204X2003001000002.

BRACKMANN, A. et al. Armazenamento de ameixas 'Reubennel' e 'Pluma 7' em frigoconservação intermitente e atmosfera controlada. Revista Científica Rural, Bagé, v.6, n.1, p.7176, 2001. Disponível em: <http://www.ufpel.tche.br/faem/ agrociencia/ v11n1/artigo14.pdf>. Acesso em: 22 set. 2009.

BRACKMANN, A. et al. Qualidade da maçã cv. 'Gala' tratada com 1-metilciclopropeno. Ciência Rural, Santa Maria, v.34, n.5, p.1415-1420, 2004. Disponível em: <http://www.scielo.br/ pdf/cr/v34n5/a14v34n5.pdf>. Acesso em: 22 set. 2009. doi:101590/S0103-84782004000500014.

BRACKMANN, A. et al. Indução da perda de massa fresca e a ocorrência de distúrbios fisiológicos em maçãs 'Royal Gala' durante o armazenamento em atmosfera controlada. Revista Brasileira de Armazenamento, Viçosa, v.32, n.2, p.87-92, 2007.

CANDAN, A.P. et al. Improvement of storability and shef-life of 'Blackamber' plums treated with 1-methylcyclopropene. Food Science and Technology International, London, v.12. n.5, p.437-443, 2006.

DONG, L. et al. S. Ripening of 'Red Rosa'plums: effect of ethylene and 1-metilciclopropene. Journal of Plant Physiology, Jena, n.28, p.1039-1045, 2001.
DONG, L. et al. Effect of 1- metihycyclopropene on ripening of 'Canino' apricots and 'Royal Zee' plums. Postharvest Biology and Technology, Amsterdam, n.24, p.135-145, 2002. Disponivel em: $<\mathrm{http}$ ://www.sciencedirect.com/science? ob=ArticleURL\& udi=B6TBJ456 T 3 B $5-4 \&$ \& s e r $=10 \&$ \& c ove r D a t e $=03 \% 2$ F $31 \% 2$ F $2002 \&$ alid=1021785164\& rdoc=1\&fmt $=$ high \&orig $=$ search $\&$ cdi $=5144 \&$ sort $=$ r\&_docanchor $=\& v i e w$ 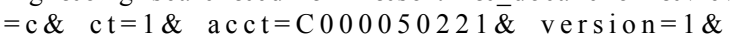 u r 1 V e r s i o n $=0 \&$ u s e r i d $=10 \&$ m d $5=$ 473c784b3994d956b1dfd472dc50 0 ecd0>. Acesso em: 22 set. 2009. doi:10.1016/S0925-5214(01)00130-2 .

JOHNSTON, J.W. et al. Temperature induces differential softening responses in apple cultivars. Postharvest Biology and Technology, Amsterdam, v.23, n.3, p.185-196, 2001. Disponível em: $<$ http://www.sciencedirect.com/ science? ob=ArticleURL\& udi=B6TBJ-44BM6WY$2 \&$ user $=10$ \& coverDate $=12 \% 2$ F3 $1 \% 2$ F2 $001 \&$ alid $=102178$

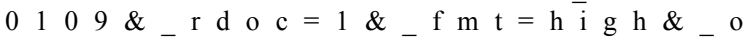
rig $=$ s e a r c h \& $\quad$ c di $=5144 \& \quad$ s ort $=$ r \& doca n c hor $=\&$ view $=\mathrm{c} \&$ ct $=1 \&$ acct $=\overline{\mathrm{C}} 000050221 \&$ versio $\mathrm{n}=1 \&$ u r 1 Ve r s i o $\mathrm{n}=0 \&$ u s e r i d = $10 \&$ md5 $=9 \mathrm{e} 231995213 \mathrm{c} 8252 \mathrm{bdeaa} 5396 \mathrm{c} 5 \mathrm{eeled} \gg$. Acesso em: 19 set. 2009. doi:10.1016/S0925-5214(01)00127-2 .

MAJUMDER, K.; MAZUMDAR, B.C. Changes of pectic substances in developing fruits of cape-gooseberry (Physalis peruviana L.) in relation to the enzyme activity and evolution of ethylene. Scientia Horticulturae, Amsterdam, v.96, n.1-4, p.91-101, 2002. Disponivel em: <http://www.sciencedirect.com/ science? ob=ArticleURL \& udi=B6TC3-45Y6H6T$2 \&$ _user $=10 \&$ _coverDate $=12 \% 2 \mathrm{~F} 06 \% 2 \mathrm{~F} 2002 \&$ alid $=1$ $021807322 \&$ rdoc $=1 \&$ fmt $=$ high\&_orig $=$ search\&_cdi $=515$ $9 \&$ sort $=$ r \& docanchor $=\&$ view $=$ c\& ct $=1 \&$ acct $=\mathrm{C}$ 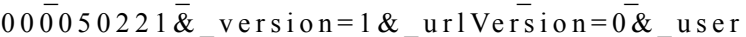 $\mathrm{id}=10 \& \mathrm{md} 5=$ adafba112ccda5fd9c2741f9c4179fb4>. Acesso em: 22 set. 2009. doi:10.1016/S0304-4238(02)00079-1

MANGANARIS, G.A. et al. Novel 1-metihycyclopropene immersion formulation extends shelf life of advanced maturity 'Joanna Red' plums (Prunus salicina Lindell). Postharvest Biology and Technology, Amsterdam, v.47, p. 429-433, 2008. Disponível em: <http://www.sciencedirect.com/ science? ob=ArticleURL\& udi=B6TBJ-4PMYXH 5$2 \&$ user $=10 \&$ \&coverDate $=03 \% 2 \mathrm{~F} 31 \% 2 \mathrm{~F} 2008 \&$ alid $=1021805587 \&$ rd o $\bar{c}=1 \& \&_{-} \mathrm{m} \mathrm{t}^{-} \mathrm{h}$ i g h \& $\&_{-}$r i $\bar{g}=\mathrm{s}$ e a r c h \& c di $=5144 \&$ s ort $=$ r \&_doc a n chor $=\&$ vi e w $=\bar{c}$ $\&_{-} \mathrm{ct}=1 \&$ \& a c t $=$ C $000050221 \&$ \&version $=1 \&$ \& ur 1 $\mathrm{V}$ e r s i o $\mathrm{n}=0 \&$ u s e r i d $=10 \& \mathrm{~m} \mathrm{~d} 5=6 \mathrm{a}$ 666bf6b872e8f05e3f28716a579ec5>. Acesso em: 22 set. 2009. doi:10.1016/j.postharvbio.2007.07.006.

MENNITI, A.M. et al. 1-Methylcyclopropene retards postharvest softening of plums. Postharvest Biology and Technology, Amsterdam, v.31, p.269-275, 2004. Disponivel em: $<$ http://www.sciencedirect.com/ science?_ob=ArticleURL\&_udi=B6TBJ-4BRP 586 $4 \&$ user $=10 \&$ coverDate $=03 \% 2 \mathrm{~F} 31 \% 2 \mathrm{~F} 2004 \&$ alid $=1021793604$ \&_rdoc $=1 \&$ fmt $=$ high\&_orig $=$ search\&_cdi $=5144 \&$ sort $=r$ $\&$ docanchor $=\&$ view $=$ c \& ct $=1 \&$ acct $=$ C 00005022 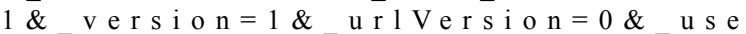 rid $=1 \overline{0} \& \mathrm{md} 5=\mathrm{af} 74 \mathrm{dbfb} 88 \mathrm{bfe} 1 \overline{4} 723 \mathrm{c} 4 \mathrm{bc} 4 \mathrm{~d} 09 \mathrm{fc} 5 \mathrm{aeb}>$. Acesso em: 22 set 2009. doi:10.1016/j.postharvbio.2003.09.009.

STEFFENS, C.A. et al. Taxa respiratória de frutas de clima temperado. Pesquisa Agropecuária Brasileira, Brasília, v.42, n.3, p.313-321, 2007. Disponível em: <http:// w w w. s c i e 1 o.b r/s c i e 1 o.ph p ? p id=S $0100-$ 
204X2007000300003\&script=sci_arttext $>$. Acesso em: 22 set. 2009. doi: 10.1590/S0100-204X2007000300003.

VALERO, D. et al. Quality improvement and extension of shelf life by 1-methylcyclopropene in plum as affected by ripening stage at harvest. Innovative Food Science \& Emerging Technologies, Amsterdam, v.4, n.3, p.339-348, 2003. Disponível em: <http://www.sciencedirect.com/ science? ob=ArticleURL\& udi=B6W6D-49G4498$2 \&$ user $=10 \&$ coverDate $=09 \% 2 \mathrm{~F} 30 \% 2 \mathrm{~F} 2003 \&$ alid $=1021801$ $670 \&$ rdoc $=1 \&$ fmt $=$ high\&_orig $=$ search\&_cdi $=6596 \&$ sort $=r \&$ docanchor $=\& v i e w=c \&$ ct $=1 \&$ \& acct $=$ C 00005022 $1 \&$ version $=1 \&$ u r 1 Version $=0 \&$ useri $\mathrm{d}=10 \& \mathrm{md} 5=4249 \mathrm{a} 286 \mathrm{dbb} 34715 \mathrm{~b} 7215723 \mathrm{fb} 6152 \mathrm{~d} 4>$. Acesso em: 22 set 2009. doi:10.1016/S1466-8564(03)00038-9.

VALERO, D. et al. Could the 1-MCP treatment effectiveness in plum be affected by packaging? Postharvest Biology and Technology, Amsterdam, v.34, p.295-303, 2004. Disponivel em: $<\mathrm{h} \mathrm{t} \mathrm{t} \mathrm{p} \mathrm{:} \mathrm{//} \mathrm{w} \mathrm{w} \mathrm{w.} \mathrm{s} \mathrm{c} \mathrm{i} \mathrm{e} \mathrm{n} \mathrm{c} \mathrm{e} \mathrm{d} \mathrm{i} \mathrm{rect.} \mathrm{co} \mathrm{m/}$ science? ob=ArticleURL\& udi=B6TBJ-4DCWGXF$3 \&$ user $=10 \&$ _coverDate $=12 \% 2 \mathrm{~F} 31 \% 2 \mathrm{~F} 2004 \&$ _alid $=10$ $21795232 \&$ rdoc $=1 \&$ fmt $=$ high\&_orig $=$ search\&_cdi $=5144 \&$ do canchor $=\&$ view $=c \&$ ct $=1 \&$ \& acct $=C 000050221 \&$ \& version $=$ $1 \&$ urlVersion $=0 \&$ userid $=10 \& \mathrm{~m} \mathrm{~d} 5=\mathrm{e} 595 \mathrm{dbbf0} 1 \mathrm{f} 1082 \mathrm{~b} 5 \mathrm{~b} 749 \mathrm{a}$ 188aaae320>. Acesso em 22 set. de 2009. doi:10.1016/ j.postharvbio.2004.05.020

WATKINS, C.B. The use of 1-methylcyclopropene (1-MCP) on fruits and vegetables. Biotechnology Advances, Amsterdam, n.24, p.389-409, 2006. Disponível em: <http://www.sciencedirect.com/ science? ob=ArticleURL\& udi=B6T4X-4JFGFB 4 $1 \&$ _user $=10 \&$ _coverDate $=08 \% 2 \mathrm{~F} 31 \% 2 \mathrm{~F} 2006 \&$ alid $=10$ $21802930 \&$ rdoc $=1 \&$ fmt $=$ high\& orig $=$ search \& cdi $=4986$ \&_sort $=$ r\&_docanchor $=\& v i e w=c \& \_c t=1 \& \_a c c t=C 0000$ $50221 \&$ \& version $=1 \&$ ur 1 Version $=0 \&$ \& user id $=10 \& m d 5=$ fc $363 e 6 e 31793 f 495 b 0367 \mathrm{a} 853 \mathrm{f} 67 \mathrm{ef0}>$. Acesso em: 22 set. 2009. doi:10.1016/j.biotechadv.2006.01.005. 